\title{
Molecular Detection of Rupestris stem pitting-associated virus in Grapevines in Brazil
}

\author{
Luciana M. Espinha ${ }^{1 *}$, José O. Gaspar ${ }^{1}$, H. Kuniyuki ${ }^{2 * *} \&$ Luis E. A. Camargo ${ }^{3 * *}$ \\ ${ }^{1}$ IBILCE/UNESP, CEP 15054-000, São José do Rio Preto, SP, ${ }^{2}$ IAC, Centro de Fitossanidade, CEP 13020-902, Campinas, \\ SP; ${ }^{3}$ ESALQ-USP, Cx. Postal 9, CEP 13418-900, Piracicaba, SP
}

(Aceito para publicação em 12/03/2003)

Autor para correspondência: José Osmar Gaspar

\section{RESUMO}

Detecção por métodos moleculares do Rupestris stem pittingassociated virus em videiras no Brasil

RT-PCR foi utilizada para amplificar parte do gene da replicase do Rupestris stem pitting-associated virus (RSPaV) a partir de videiras (Vitis spp.) no Brasil. O segmento amplificado foi clonado e seqüenciado e, por comparação da seqüência de nucleotídeos e dos aminoácidos deduzidos, verificou-se que correspondiam, respectivamente, em $88 \%$ e $94 \%$ com as de isolados do RSPaV de outros países.
Rupestris stem pitting (RSP), a component of the rugose wood complex, is one of the most widespread grafttransmissible grapevines (Vitis spp.) virus. It was detected in Brazil late in the 60's (Kuniyuki. Fitopatol. Bras. 5:137, 1972). The RSP is characterized by the presence of small pits in the woody cylinder below the point of inoculation by chip budding on Vitis rupestris Scheele cv. St. George ('du Lot') (Goheen. Compendium of Grape Diseases. 1988. p.53). A virus named Ruspestris stem pitting-associated virus (RSPaV), Foveavirus genus, has been associated with the disease (Zhang et al. Phytopathology 88:1231, 1998; Meng et al. Eur. J. Plant Pathol. 105:191, 1999). This work reports the molecular detection of RSPaV in grapevines by RT/PCR, nucleotide sequence analysis and a non-isotopic cDNA probe specific to RSPaV. The RNA was extracted from petioles and used in the RT-PCR reaction with RSPaV-specific primers (Zhang et al. Phytopathology 88:1231, 1998). The amplified fragment was cloned and sequenced. The nucleotide $(88 \%)$ and deduced amino acid (94\%) sequences of the gene fragment (831 pb; Figure 1A) showed high homologies with those of two other RSPaV isolates (GenBank AF6278; AF057136). A digoxigenin-labeled cDNA probe was generated by PCR and used to positively detect RSPaV in RNA extracted from varieties of $V$. vinifera $\mathrm{L}$. (Figure 1B). These results confirm the presence of RSPaV in grapevines that had indexed positive for RSP on V. rupestris 'St. George' in Brazil. The RT-PCR technique together with the cDNA probe will be a useful procedure for rapidly detecting the RSP disease in grapevines.

Financial support: FAPESP, CAPES

*CAPES fellowship; ** CNPq fellowship
A

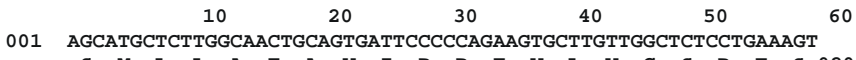


CTAAATCCTTGGGCCTACCAGTACAGAATTAGTGATAATCAACTGCTCTTCGCACCTGAT $\begin{array}{lllllllllllllllllllll}\mathbf{L} & \mathbf{N} & \mathbf{P} & \mathbf{W} & \mathbf{A} & \mathbf{Y} & \mathbf{Q} & \mathbf{Y} & \mathbf{R} & \mathbf{I} & \mathbf{S} & \mathbf{D} & \mathbf{N} & \mathbf{Q} & \mathbf{L} & \mathbf{L} & \mathbf{F} & \mathbf{A} & \mathbf{P} & \mathbf{D} & 040\end{array}$ GGTAATTGGAGTGAAATGTATTCACAGCCTTTGTCATGCAGATACCTACTTAAGGCTAGA

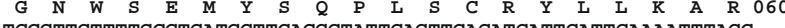
TCCGTTGTTTTGCCTGATGGTTCACGCTATTCAGTTGACATCATTCATTCAAAATTTAGC

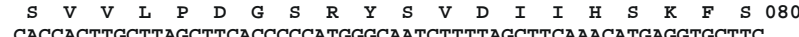

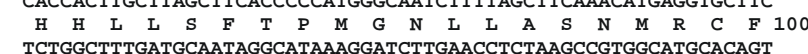
TCTGGCTTTGATGCAATAGGCATAAAGGATCTTGAACCTCTAAGCCGTGGCATGCACAGT

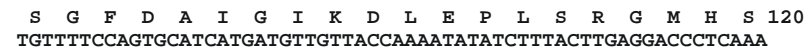
TGTTTTCCAGTGCATCATGATGTTGTTACCAAAATATATCTTTACTTGAGGACCCTCAAA $\begin{array}{lllllllllllllllllllll}C & F & P & V & H & H & D & V & V & T & K & I & Y & \text { L } & \text { Y } & \text { L } & R & \text { T } & \text { L } & K & 140\end{array}$ AAGCCAGACAAGGAGTCTGCAGAGGCAAAGCTTCGTCAACTCATTGATAAGCCCACAGGG $\begin{array}{lllllllllllllllllllll}K & \mathbf{P} & \mathbf{D} & \mathbf{K} & \mathbf{E} & \mathbf{S} & \mathbf{A} & \mathbf{E} & \mathbf{A} & \mathrm{K} & \mathrm{L} & \mathbf{R} & \mathbf{Q} & \mathrm{I} & \mathrm{I} & \mathbf{D} & \mathbf{K} & \mathbf{P} & \mathbf{T} & \mathbf{G} & 160\end{array}$ AGGGAGATAAAATTCATTGAAGATTTTTCCTCACTAGTTATAAGTTGTGGAAGGAGTGGT $\begin{array}{llllllllllllllllllllll}R & E & I & K & F & I & E & D & F & S & S & \text { L } & \text { V } & \text { I } & \text { S } & \text { C } & \text { G } & R & \text { S } & \text { G } & 180\end{array}$ TCTTTGCTTATGCCCAACATTTCTAAGTTGGTAATATCATTCTTTTGCCGAATGATGCCA $\begin{array}{lllllllllllllllllllllll}\text { S } & \text { L } & \text { L } & \text { M } & \text { P } & \text { N } & \text { I } & \text { S } & K & \text { L } & \text { V } & \text { I } & \text { S } & \text { F } & \text { F } & \text { C } & \text { R } & \text { M } & \text { M } & \text { P } & 200\end{array}$ AATGCACTTGCTAGGCTTTCTTCCAATTTTCGGGAGTGCTCACTGGATTCATTTGTGTAT $\begin{array}{lllllllllllllllllllllll}\text { N } & \text { A } & \text { L } & A & R & \text { L } & \text { S } & \text { S } & \text { N } & \text { F } & R & \text { E } & \text { C } & \text { S } & \text { L } & \text { D } & \text { S } & \text { F } & \text { V } & \text { Y } & 220\end{array}$ TCACTTGAGCCTTTCAATTTTTCAATTAATTTGGTGGATATCACTCCCGATTTCTTTGAG $\begin{array}{lllllllllllllllllllll}S & L & E & P & F & \text { N } & F & S & I & \text { N } & \text { L } & \text { V } & \text { D } & \text { I } & \text { T } & \text { P } & \text { D } & \text { F } & \text { F } & \text { E } & 240\end{array}$ CATTTATTTCTTTTCTCTTGTCTCAATGAGTTAATCGAGGAGAATGTTGAAGAGGTCATG $\begin{array}{lllllllllllllllllllll}\text { H } & \text { L } & \text { F } & \text { L } & \text { F } & \text { S } & \text { C } & \text { L } & \text { N } & \text { E } & \text { L } & \text { I } & \text { E } & \text { E } & \text { N } & \text { V } & \text { E } & \text { E } & \text { V } & \text { M } & 260\end{array}$

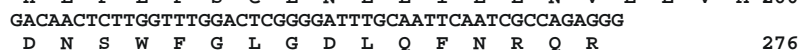
276

$\mathbf{B}$

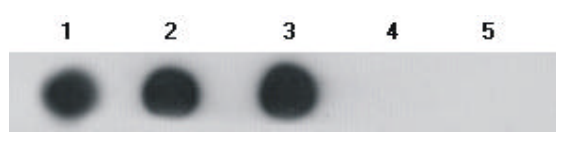

FIG. 1 - (A) Partial nucleotide (above) and deduced amino acid (below) sequences of Rupestris stem pitting-associated virus (RSPaV). (B) Dot-blot hybridization with RSPaV-specific probe. RNA extracted from infected plants of Vitis vinifera cultivars: (1) Itália (Biritiba Mirim, SP); (2) Itália (São Miguel Arcanjo, SP); and (3) Benitaka (Londrina, PR). RNA extracted from healthy plants of 'Itália' (4) and 'Benitaka' (5). 